\title{
Medico-legal implications of Neonatal Cerebral Palsy and the responsibilities of modern Obstetric-Neonatal Unit
}

\author{
George Gregory Buttigieg KM* \\ Senior Obstetrician and Gynecologist, University of Malta, Europe
}

Received: 此 May 01, 2018; Published: 畊 May 17, 2018

*Corresponding author: George Gregory Buttigieg, MD, LRCP(Eng.), MRCS(Lond.), MA(Melit.), Dip.FP, FRCOG, FRCPI, Senior Obstetrician and Gynecologist, University of Malta, Europe

\section{Editorial}

Modern medico-legal evaluation of Neonatal Cerebral Palsy (NCP) demands recognition of Neonatal Hypoxic Ischaemic Encephalopathy (NHIE) as an indispensable proof of peri-partum fetal hypoxia. Without proven NHIE, modern jurisprudence should not even consider obstetric/paediatric liability of causative peri-partum hypoxia of negligence on the grounds of medically responsible fetal/newborn hypoxia leading to Cerebral Palsy. Peripartum hypoxia, which comprises intra-partum hypoxia may result in damage which may with varying degrees of time manifest itself with epilepsy, neuro-developmental delay, cognitive impairment or Cerebral Palsy and the full damage may not be completely assessable before 3-4 years of age. Court cases alleging obstetric/ neonatologist liability may not commence for a number of years, at times even decades after he birth. In the case of Cerebral Palsy, it must be borne in mind that peri-partum hypoxia is not, nowadays, considered as causative in more than $9 \%$ of cases, whereas $91 \%$ may be due to premature birth, other complications or of undetermined aetiology. Since in many cases, CP litigation may reach Court without screening as to what may be liable from peripartum hypoxia or otherwise, it is crucial that in the hours or days post-birth, all relevant investigations are performed, stored safely and be accessible if and when necessary.

It is therefore crucial for the modern birthing Unit, to be conscious of cases where CP is evident or suspected and proceed on an established protocol of action. The usual and universal Apgar scoring, intra-partum cardio-tocographic tracing as well as fetal intra- or peri-partum acid-base and oxygenation status are a must do, but not sufficient investigations, as discussed later.

The $21^{\text {st }}$ obstetric/neonatal unit needs must be fully conscious of the medico-legal implications of cases of NCP where causation such as peri-partum hypoxia may, in the future, be cited for basis of medical liability. Where NCP is suspected, all medical/nursing staff must be conscious of a) The early presentation of the condition.

b) Underlying causation with special reference to NHIE.

c) The criteria of establishing of NHIE.

d) Modern management of NHIE.

The main scope of this Editorial is to stress the responsibility of the modern birthing Unit with special reference to point three. This is crucial for many reasons, chief of which is the commencement of the right management of NCP. A high degree of suspicion of the potential of the condition must be kept in situations where severe maternal hypotension, especially if protracted (such as in maternal blood loss, abruption placentae, epidural/postural induced hypotension, uterine rupture, coagulopathies ,cardiac complications....) or in proven or likely potential fetal compromise as in umbilical cord prolapse, intra-partum haemorrhage, trauma, genuine fetal distress....).

In the rush of caring for the neonate, it is all too easy that a serious Court case may erupt a decade later. This demands a number of points of management starting with the full recording and timing of all events which includes at what time which doctor/specialist was contacted and his response action. The careful storage of all CTG tracings is a must. The ensuring of the highest hierarchy of obstetric/pediatric care is crucial and as far as investigations go, one must go further than the routine and established norm. Fetal brain MRI and the newer methods such as MRI Spectroscopy and diffusion weighted imaging at the right time may go a long way in throwing the right light for justice to supervene if and when litigation arises. These techniques do not only demand specialised equipment but also specialised personnel to perform and interpret such advanced techniques. These investigations may be looked askance in some Units at the moment, but, a time may come, when their omission may carry its own liability. 
As a final point, one must also remember that the personnel of the modern birthing Unit, should be well versed in the modern medico-legal concepts in which patient and parental autonomy are forever gaining momentum at Court. It is crucial to maintain the parents fully aware of what investigations are being suggested and why. Communication with the child's parents should be held by the most senior and not the most junior of the caring team. Such discussions must now sow the wrong seeds, especially in generating suggestions of liability in a subject, which has for decades, suffered from intra-partum hypoxia being wrongly labelled as causative of CP. On the other hand, one must be precise and objective in one's discussions. Ruling out peri-partum hypoxia from the latest sophisticated investigations does not rule out liability from other aspects of obstetric management. Vice-versa, confirming hypoxia and NHIE as likely causative factors of CP is not an ipso facto determinant of liability.
This work is licensed under Creative Commons Attribution 4.0 License

To Submit Your Article Click Here:

Submit Article
DOI: $10.32474 /$ PAPN.2018.01.000118

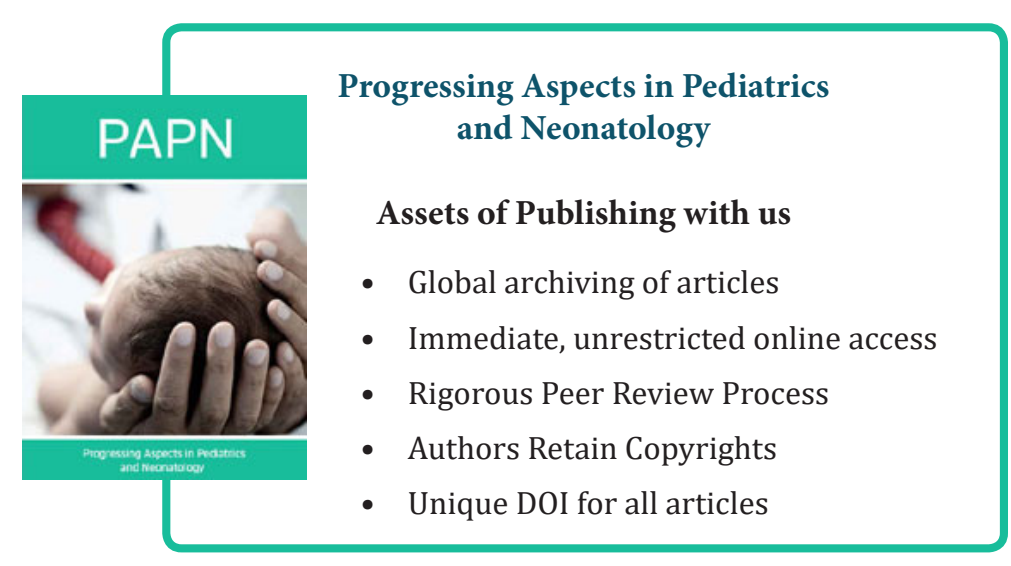

